Revue des patrimoines

\title{
Imagerie numérique et conservation-restauration : état de l'art et perspectives
}

Digital imaging and conservation-restoration

Roland May, Odile Guillon, Anthony Pamart et Jean-Marc Vallet

\section{CpenEdition}

\section{Journals}

Édition électronique

URL : http://journals.openedition.org/insitu/21940

DOI : $10.4000 /$ insitu. 21940

ISSN : 1630-7305

Éditeur

Ministère de la Culture

Référence électronique

Roland May, Odile Guillon, Anthony Pamart et Jean-Marc Vallet, « Imagerie numérique et

conservation-restauration : état de l'art et perspectives », In Situ [En ligne], 39 | 2019, mis en ligne le 31 mai 2019, consulté le 10 décembre 2020. URL : http://journals.openedition.org/insitu/21940 ; DOI https://doi.org/10.4000/insitu.21940

Ce document a été généré automatiquement le 10 décembre 2020.

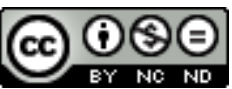

In Situ Revues des patrimoines est mis à disposition selon les termes de la licence Creative Commons Attribution - Pas d'Utilisation Commerciale - Pas de Modification 4.0 International. 


\title{
Imagerie numérique et conservation-restauration : état de l'art et perspectives
}

\author{
Digital imaging and conservation-restoration
}

Roland May, Odile Guillon, Anthony Pamart et Jean-Marc Vallet

1 L'accès à l'invisible, l'identification visuelle, l'apport documentaire ont fait de l'imagerie scientifique (dite aussi imagerie technique, technical imaging) un outil majeur dans l'apport des sciences à la conservation-restauration dans la première moitié $\mathrm{du} \mathrm{xx}^{\mathrm{e}}$ siècle. Outil de connaissance et traceur de l'histoire matérielle, elle a modifié considérablement les conditions d'intervention sur les œuvres en apportant une aide précieuse lors des différentes étapes d'un projet de restauration.

2 Depuis une trentaine d'années, la place et l'apport de l'imagerie technique numérique en conservation ont connu une mutation profonde. Simple composante - illustrée - d'un travail, d'une étude, elle est devenue une référence à part entière, au même titre que les analyses physico-chimiques, le constat ou le diagnostic et s'est imposée comme l'outil d'investigation non destructif par excellence. D'autre part, les progrès en imagerie scientifique, cumulés à l'essor des techniques de numérisation 3D, sont à l'origine de nouvelles méthodes d'analyse pour la conservation préventive, curative et la restauration du patrimoine culturel ${ }^{1}$.

3 Cet article propose de relater cette évolution et d'évoquer des pistes de réflexion basées notamment sur les travaux récents du laboratoire commun établi entre le CICRP (Centre interdisciplinaire de conservation et de restauration du patrimoine, Marseille) et l'UMR MAP (Modèles et simulations pour l'architecture et le patrimoine) (infra), enrichissant mutuellement ces méthodologies d'études pour que l'objet numérique à patrimonial devienne un nouveau vecteur de connaissance. 


\section{De l'apport de l'imagerie numérique à la documentation scientifique pour l'étude des biens culturels}

4 À la différence de la photographie scientifique réalisée sur support argentique, les nouvelles technologies numériques bénéficient, dans le domaine de l'imagerie technique, - apparemment - d'une accessibilité accrue, technique et financière. Elles permettent aux acteurs de la conservation-restauration un examen approfondi de ces images à vocation documentaire et scientifique, offrant un support visuel et un complément d'information pour retracer et enrichir l'histoire d'une œuvre².

5 Ces apports ont non seulement suscité une réflexion sur les protocoles d'acquisition et de traitement d'images mais aussi sur le regard porté aux documents eux-mêmes. Grâce à une réponse quasi instantanée et possiblement modifiable, cette distance critique incite à aller au-delà d'une simple lecture de premier résultat. L'imagerie digitale amène, par sa facilité de maniement, à explorer, analyser beaucoup plus finement les modalités de prise de vue, tant les interactions possibles offrent un vaste «terrain de jeu»: lecture sur écran et possibilité de se déplacer sur la totalité de l'image à de forts agrandissements, manipulations des histogrammes et des trois couches Rouge-Vert-Bleu (RVB) de l'image, superposition ou fusion des acquisitions par la création de calques, etc. Malgré une complexité accrue de la technologie des boîtiers numériques et le développement de logiciels de traitement de plus en plus perfectionnés et souvent opaques, réinventer les protocoles d'acquisition et de traitement issus de l'analogique a été le premier grand chantier de cette révolution. Le virage numérique a invité les photographes du patrimoine à adapter les méthodes et les routines à ce nouveau support, à se les approprier, à les maîtriser autant que possible tout en déjouant et en s'affranchissant des automatismes "embellisseur d'images" développés par les concepteurs. Du déclenchement à distance en studio ou sur le terrain à la retouche assistée par tablette graphique, le digital a modifié les pratiques (fig. 1). 
Figure 1

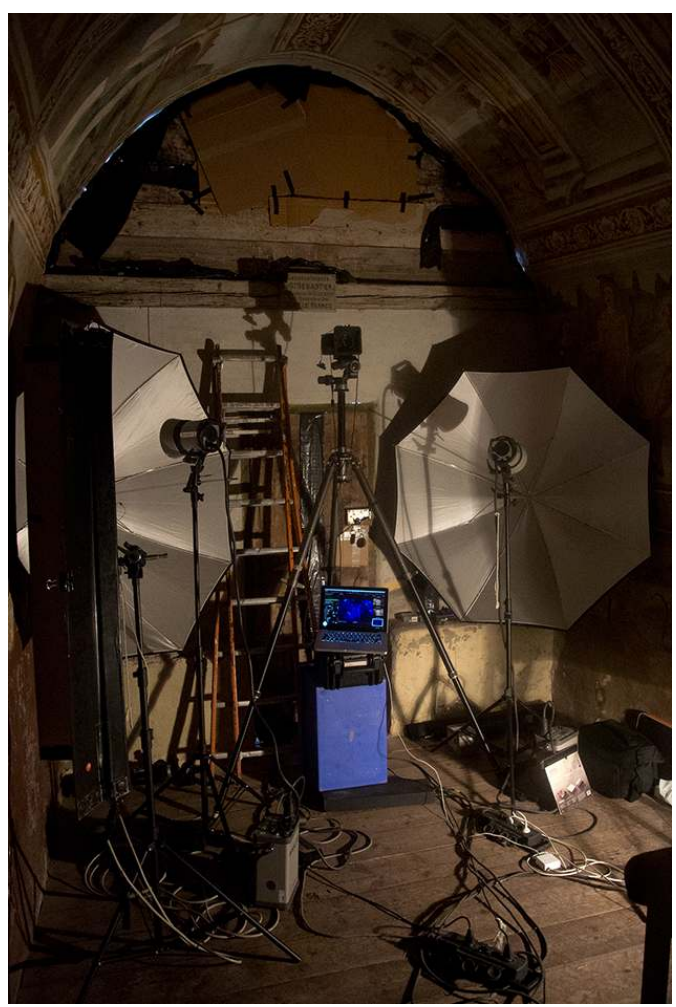

Installation pour une acquisition d'images à la chapelle Saint-Sébastien-et-Saint-Bernard à Roure (Alpes-Maritimes).

Phot. Odile Guillon, 2013. (c) CICRP.

6 Le résultat immédiat, grâce au l'affichage et à la possible correction du fichier in situ, ont mis également un terme à l'angoisse de la découverte d'une campagne ratée lors du développement des films. L'acquisition en format RAW (considéré comme le négatif numérique) permet à tout moment de revenir sur le traitement de l'image initiale. L'imagerie couleur est dans une certaine mesure mieux maîtrisée grâce entre autres à un calibrage plus précis de la température de couleur. Le rendu des couleurs s'en trouve amélioré et restitue une vision plus conforme à la réalité. Les protocoles d'acquisition et de traitement sont facilement reproductibles grâce à la création de processus informatisés. Les jeux de réglages sur l'image sont infinis et permettent de générer de nombreuses variantes de la même image en termes de densité, de contraste, de couleur... Ces interprétations peuvent être réalisées sur l'intégralité de l'image comme sur des zones préalablement sélectionnées par la création de masques et de calques de réglage spécifiques à cette sélection. Tout cela n'est qu'une infime partie des possibilités qu'offre la technologie numérique.

7 Ces multiples et nouvelles capacités obligent les professionnels de l'image à se tenir à une veille technologique constante et approfondie, à s'intéresser à d'autres technologies de capteurs et à suivre leur évolution, leur maturation technique. Il est désormais possible et même recommandé d'adapter son matériel aux conditions et objectifs du relevé. En effet, tout comme l'on adaptait la pellicule et la chimie de développement, il n'est pas systématiquement nécessaire de choisir une haute densité de pixels (valeur en mégapixel) dans le cas de conditions d'éclairage insatisfaisantes, il convient plutôt de favoriser un 
capteur dont les photosites plus larges amélioreront la qualité de réception du signal lumineux. Ainsi, la sensibilité des capteurs permet dorénavant de mettre en œuvre des acquisitions peu utilisées en analogique en raison de temps de pose très longs, pouvant atteindre plusieurs heures d'exposition, telle que l'image couleur de fluorescence sous ultraviolet $^{3}$ (fig. 2).

Figure 2

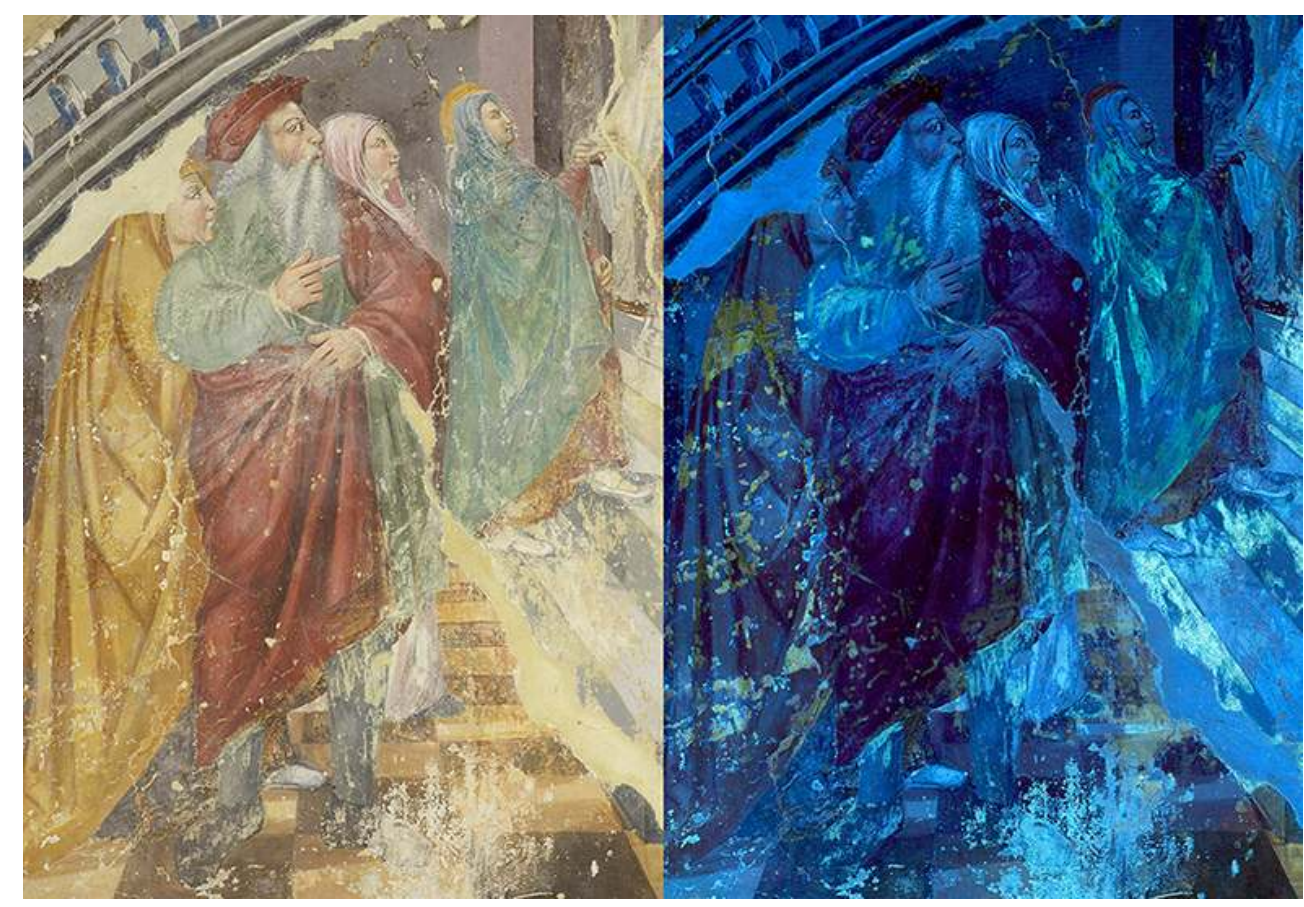

(a,b) Détail du mur nord de la chapelle Notre-Dame-d'Entrevignes à Sigale (Alpes-Maritimes) ; a-Image en lumière directe (à gauche), b-Image de fluorescence sous rayonnement ultraviolet (à droite).

Phot. Odile Guillon, 2013. (c) CICRP.

En contrepartie, la technicité accrue de ces pratiques apporte son lot de problématiques pixels morts, gestion des profils colorimétriques complexe, interprétation du capteur non maitrisable - ou d'imperfections - bruit informatique en faible luminosité (points colorés affectant particulièrement les zones sombres), aberrations chromatiques, artefacts dans l'imagerie infrarouge (IR)... Ces limites nous interrogent constamment sur l'obsolescence du matériel face à la fragilité et au développement des capteurs (2 millions de pixels en 1990 et au-delà de 100 millions de pixels actuellement), sur la pérennité présumée de l'image numérique, tout ceci non sans un impact financier important. Malgré un net progrès en termes de définition et de résolution, ces défauts de l'image numérique - du pixel jusqu'à sa composition - ont imposé une remise en cause de sa valeur informative et analytique. Ce questionnement s'inscrit dans la réflexion plus générale sur le caractère instable et variable propre à l'image photographique numérique (fig. 3). 
Figure 3

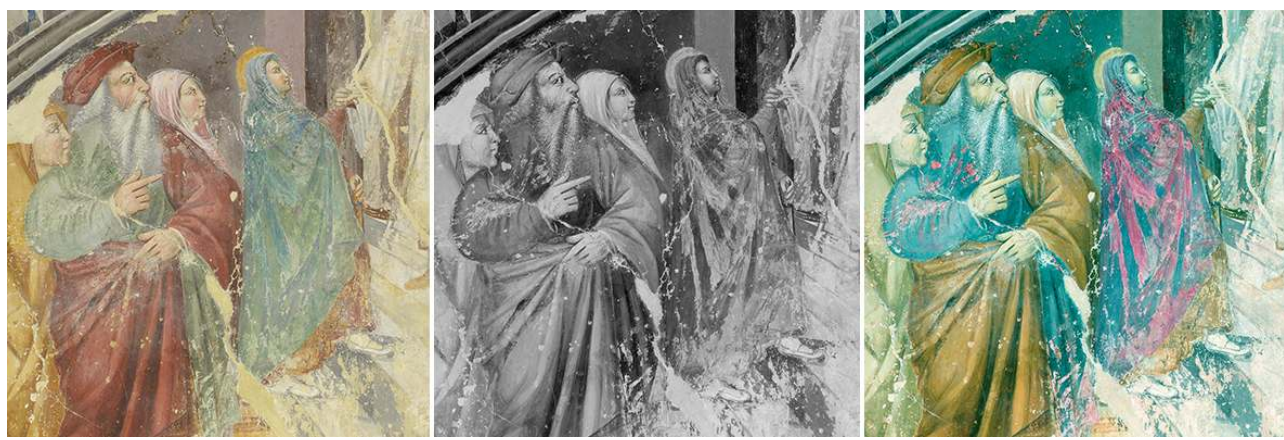

$(a, b, c)$ Détail du mur nord de la chapelle Notre-Dame-d'Entrevignes à Sigale (Alpes-Maritimes). De gauche à droite : a-Image en lumière directe, b-Image dans le proche infrarouge, c-Image composite infrarouge fausse couleur.

Phot. Odile Guillon, 2013. ㄷ CICRP.

9 Cette révolution «du grain d'argent au pixel, de l'agrandisseur à l'écran » touche également le traitement, deuxième phase du processus de création d'image. Les « logiciels de traitement » incitent à aborder la transformation et l'interprétation de l'image, peu exploitées en analogique. Le numérique a grandement facilité les modifications de l'image brute pour extraire ou révéler une information latente. L'exemple le plus éloquent concerne la création d'images composites, telle que l'image conventionnellement appelée « infrarouge fausse couleur» combinant les couches rouge et verte de l'image visible «lumière directe » et une couche de l'image dans le proche infrarouge (image réalisée avec un capteur modifié sans filtre IR coupant). Cette image améliore la lecture de l'image infrarouge et facilite la réalisation de cartographie des matériaux.

10 L'image devient aussi un support d'investigation comme le montre ce travail de «dé corrélation » des 3 couches (RVB) d'une image dans le visible, manipulation qui aurait demandé de nombreuses heures voire jours de travail en analogique (fig. 4).

Figure 4

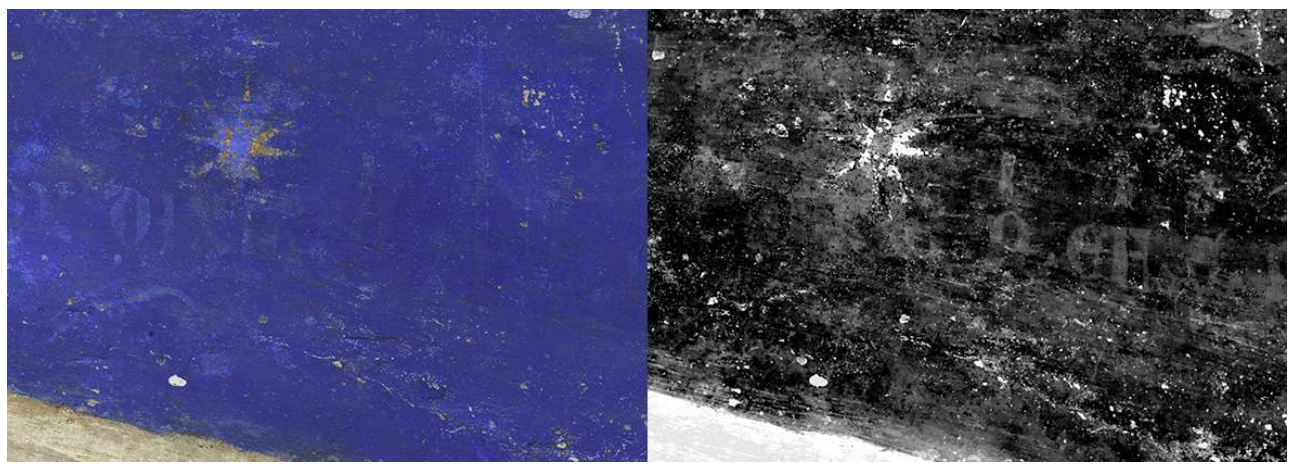

(a,b) Détail du plafond de la chapelle Saint-Martial, palais des Papes, Avignon (Vaucluse) ; a-Image en lumière directe (à gauche), b-Image modifiée (à droite).

Phot. Odile Guillon, 2013. (C) CICRP.

11 Les professionnels de la conservation ont maintenant la possibilité de travailler avec une documentation par imagerie précise, fournie pour partie par des professionnels de l'image et pour partie directement par eux-mêmes. Les photographes du patrimoine peuvent constituer des dossiers d'imagerie technique en très haute résolution, sous 
divers rayonnements, de l'UV à l'IR, et créer des images composites. Ils peuvent combiner ces images (ex. images mosaïque, IR fausse couleur, UV fausse couleur, fluorescence du visible vers l'infrarouge...) et utiliser les avancées en traitement d'image (ex. : logiciel DStretch) et en photographie computationnelle (High Dynamic Range Imaging (HDR), focus stacking) qui permettent de détecter et mettre en valeur des particularités peu visibles voire invisibles du bien culturel étudié. Le numérique a aussi favorisé le rapprochement vers d'autres méthodes d'acquisition telle que la prise de mesure directe sur une orthophotographie issue de processus photogrammétrique (aspect quantitatif), ou grâce à la sublimation des reliefs de surfaces issue du ré-éclairage interactif multidirectionnel d'une acquisition Reflectance Transformation Imaging (RTI) (aspect qualitatif).

Ces méthodes d'acquisition et leur hybridation ont par ailleurs estompé la frontière entre la 2D et la 3D. Exploitant des capteurs photographiques similaires, le LABCOM, dans ses premières expérimentations, a validé l'hypothèse que ces images pouvaient se superposer parfaitement les unes aux autres, tels des calques. L'harmonisation des protocoles d'acquisition a permis de développer une fusion de ces images par un traitement photogrammétrique spécifique afin de générer une représentation 3D augmentée ou que ces images soient fusionnées sur des nuages de points - ou orthophotographies existants, donnant ainsi naissance à des dossiers d'imagerie photogrammétrique technique ${ }^{5}$ (fig. 5).

Figure 5
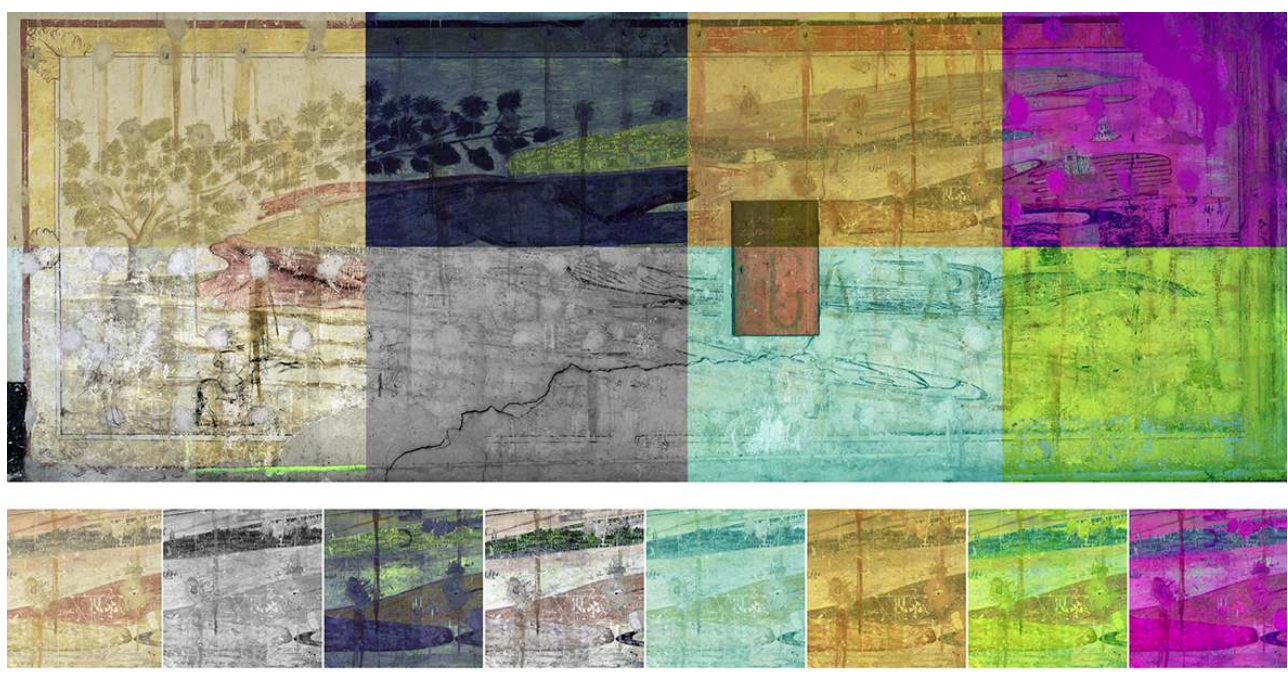

(Haut et bas) Peinture murale de la chapelle des Jésuites, Aix-en-Provence. Orthophotomosaïque multispectrale (en haut) et comparaison (en bas) des 8 modes de rendu (de gauche à droite, lumière directe, dans le proche infrarouge, fluorescence sous rayonnement UV, composite médiane, composite IR fausse couleur, composite UV fausse couleur et images composites multibandes). Phot. Odile Guillon \& Anthony Pamart, 2016. (C) Labcom CICRP-MAP.

\section{Vers une fusion multimodale des données pour la constitution d'un continuum informations 2D/3D}

13 Néanmoins, c'est probablement la mutation du statut de l'imagerie - du support documentaire au support d'analyse à contenu qui comprend en particulier la prise de 
mesure de distance, d'angle, de superficie et de volume, des annotations spatialisées, évolutives et partagées -, qui constitue l'évolution majeure en conservation-restauration.

Elle est étroitement liée, au cours de cette dernière décennie, à la photogrammétrie, autre technique d'imagerie couramment utilisée pour documenter et analyser les objets patrimoniaux. Inventée à la fin du xix siècle par Albrecht Meydenbauer pour établir des relevés précis du patrimoine architectural, son application a connu, grâce au numérique, un essor et une démocratisation considérables. Elle se base sur la multiplicité des prises de vue d'un même objet pour le reconstruire géométriquement et visuellement en une «photographie 3D » dans laquelle l'ensemble des pixels est spatialisé sous la forme d'un nuage de points. Elle crée une relation projective entre un modèle tridimensionnel et les images bidimensionnelles qui ont permis sa modélisation.

15 Dans le cadre de nos travaux et recherches appliqués au patrimoine matériel, la fusion photogrammétrique est la méthode utilisée pour spatialiser les images $2 \mathrm{D}^{6}$. Initialement employée de façon linéaire comme un moyen de générer un modèle 3D ou une orthophotographie, elle est dorénavant explorée comme un processus incrémental, permettant de référencer des images au sein d'un même système spatial. Il s'agit d'une première étape dans la fusion des données et la création d'un continuum d'informations hétérogènes, afin que l'objet numérisé puisse être exploitable comme un véritable outil d'analyse ou de suivi, voire d'exploration d'évolutions possibles.

16 Afin d'enrichir sémantiquement cette fusion 2D/3D, un système de propagation automatique d'annotations spatialisées a été développé 7 . Ces annotations effectuées directement sur une photographie sont automatiquement re-projetées sur toutes les vues 2D et 3D de l'objet. De fait, cela permet ainsi de créer des représentations hybrides, de rechercher ou de visualiser des informations 3D directement sur une image 2D. Grâce à ces avancées, il est maintenant possible d'envisager, dans un futur proche, de nouveaux outils intégrant une "gestion multidimensionnelle" des ressources numériques sur différents niveaux de perception et de représentation:

17 - un aspect «multicapteur », par le croisement des informations provenant de techniques d'imagerie très différentes (photographie argentique, radiographie, thermographie infrarouge);

18 - un aspect «multitemporel », par l'annotation du bien culturel à divers moments de son histoire matérielle, à des fins de suivi d'opération ou post-opération ;

19 - un aspect «multispectral» visant à enrichir les observations au-delà du domaine du visible ;

20 - un aspect «multiscalaire » favorisant la gestion de différentes échelles d'observation, « du macro au micro» (fig. 6). 
(a) Définition de l'annotation sur deux images
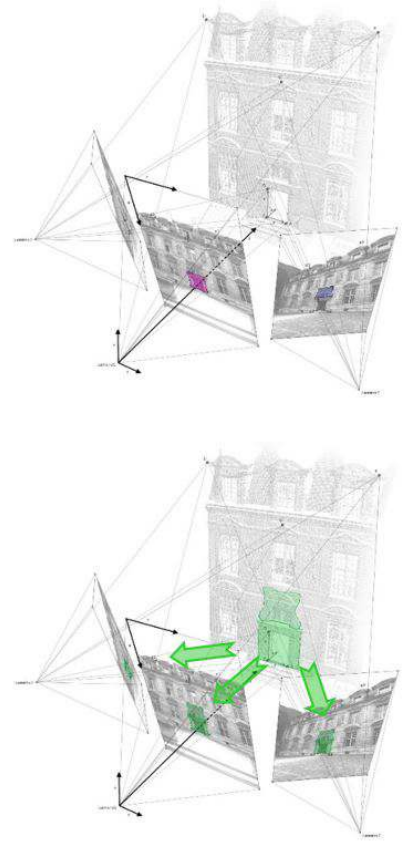

(d) Projection sur toutes les images (b) Recherche des coordonnées $3 D$ de chacune des zones

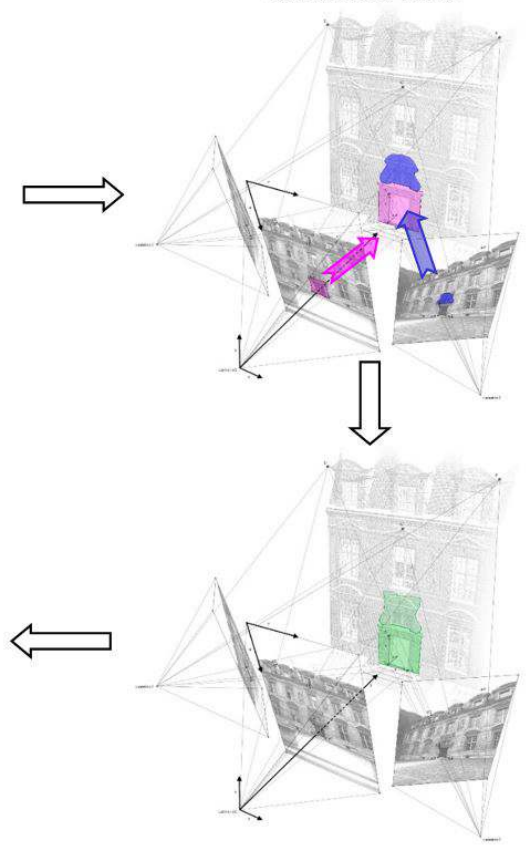

(c) Regroupement de l'ensemble des coordonnées $3 D$

Schéma de propagation automatique d'annotations sémantiques sur un jeu d'images spatialisées. Phot. Adeline Manuel, 2016. (c) UMR MAP 3495 CNRS/MCC.

La fusion de ces images techniques ouvre de nouvelles perspectives. Fusionnées ou isolées, elles constituent des instruments puissants, indispensables et complémentaires à l'analyse matérielle des biens culturels en fournissant une aide à la caractérisation des matériaux, à la localisation précise des prélèvements, à la compréhension des phénomènes de dégradation...

L'enrichissement sémantique sur la base d'annotations multidimensionnelles spatialisées permet en outre d'utiliser le support numérique pour la réalisation, par les professionnels de la conservation, de diverses cartographies qui peuvent être superposées ou combinées selon différents niveaux de lecture et de description (technique d'exécution, symptômes de dégradation, etc.). La donnée source est donc directement accessible, lorsque la fusion est techniquement possible, sur la modalité d'imagerie ayant permis l'observation (couche infrarouge, carte de profondeur...). Dans le cas inverse, elle est adjointe à une annotation relative à d'autres techniques d'analyse (microscopie 3D, coupes stratigraphiques...). Ainsi, au-delà de la restitution géométrique et visuelle de l'œuvre, la fusion 2D/3D multimodale permet d'accéder à sa matérialité par le référencement spatial d'analyses physiques et chimiques des composants de la couche picturale ${ }^{8}$. Elle ouvre le champ à leur exploration stratigraphique par des techniques d'investigation variées telles que le LIBS, l'analyse d'une coupe stratigraphique, la thermographie infrarouge (voir fig. 8). La gestion de l'ensemble de ces données dans un contexte multitemporel offre aussi de nouvelles possibilités en termes de quantification de l'évolution des phénomènes de dégradation ${ }^{9}$ (fig. 7). 


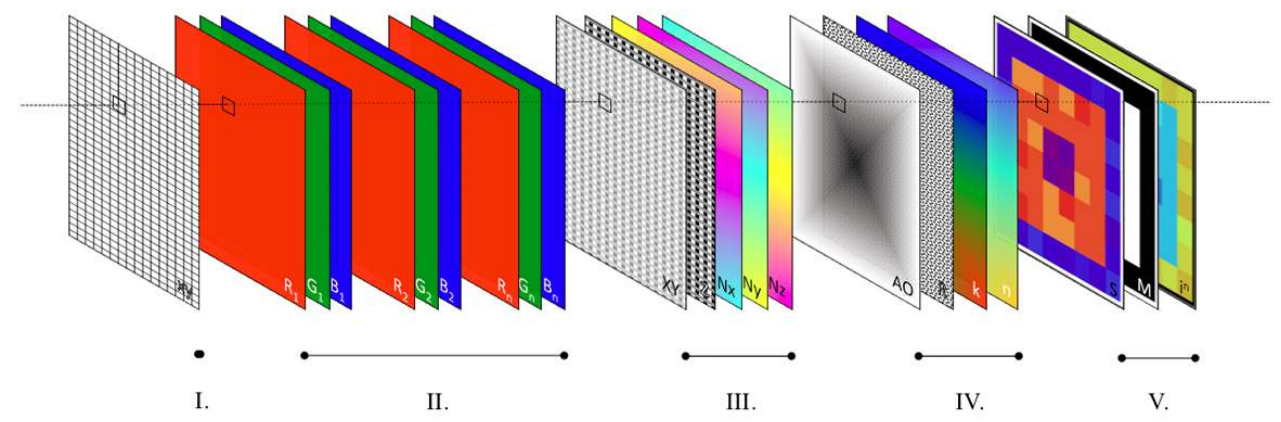

Schéma de structure du continuum d'informations multidimensionnel montrant en : i) l'indexation $2 \mathrm{D} / 3 \mathrm{D}$; ii) les valeurs colorimétriques ; iii) les propriétés géométriques ; iv) les descripteurs 2D ou 3D et en $v$ ) les multiples segmentations et annotations.

Phot. Anthony Pamart, 2016. (c) Labcom CICRP-MAP.

Support d'analyses, de connaissances et d'informations comparatives, cette nouvelle génération d'imagerie s'affirme ainsi comme un outil d'aide à la décision.

La possibilité de relier des observations à des données elles-mêmes directement spatialisées sur les images par des annotations structurées sur différents calques de description ouvre des perspectives prometteuses pour la réalisation de constats d'état, le suivi d'opérations en conservation-restauration tel que celui d'une consolidation en temps réel, apportant ainsi un support de contrôle dynamique viable.

Sous réserve de protocoles d'acquisition bien définis, la mise à disposition, l'applicabilité et la démocratisation de cette méthode de documentation accessible par une plateforme collaborative dédiée en font potentiellement un outil de dialogue entre les différents métiers de la conservation-restauration et de gestion matérielle d'un bien culturel.

\section{L'émergence de plateformes collaboratives dédiées à la conservation/restauration}

L'ouverture de telles possibilités a fait apparaitre la nécessité de développer des projets visant à mobiliser divers champs disciplinaires (l'architecture, la conservation, la mécanique, l'informatique), en concevant un prototype de chaîne de traitement de l'information. Celle-ci assure l'articulation de différentes approches, depuis la collecte de données brutes jusqu'à la production d'un savoir, en s'appuyant sur l'extraction d'informations utiles à la valorisation des connaissances apportées par les experts. Une des premières applications concernant le domaine de la conservation a porté sur le développement de la compréhension et l'analyse des phénomènes de dégradation qui affectent les édifices historiques. Elle a conduit à explorer une possibilité de dialogue interdisciplinaire et immédiat entre les professionnels de la conservation-restauration grâce à l'exploitation de l'enrichissement sémantique apporté par l'annotation directe et participative d'images spatialisées. Elle offre aussi la possibilité de tracer et de valider la pertinence des choix faits en cours d'opération, à suivre donc en temps réel un acte de restauration, voire à simuler, par modélisation, une évolution prédictive de l'état de conservation d'un bien culturel. 
27 La nature même du contenu numérique, le développement des techniques de fusion d'images, associés à l'essor des sciences des données et de l'information, sont ainsi à même de faire émerger des solutions applicatives touchant différents aspects de la conservation voire de l'histoire de l'art tels que les études stylistiques, les comparaisons d'œuvres ayant pour objectif des déterminations d'attribution, les études techniques comparées. Pour atteindre ce but, la piste suivie a été de permettre à tout expert d'enrichir les informations contenues dans une image en y associant son observation et son interprétation par le biais d'annotations sémantiques.

28 Ainsi, un premier projet, NUBES (2006-2014) ${ }^{10}$, a été développé par le MAP. Prototype d'application web pour la description, l'analyse, la documentation et le partage de représentations numériques d'édifices patrimoniaux, il combinait la photogrammétrie et les résultats issus de techniques de caractérisation classiques, sous forme de calques 3D, constituant ainsi un SIG dédié à la conservation d'un bien culturel. Une application a été développée de manière expérimentale dans le domaine de la conservation des peintures murales ${ }^{11}$. Cet outil à accessibilité limitée et conçu comme une base de données statique nécessitait un temps de travail et d'investissement important pour la réalisation d'un relevé tridimensionnel et de l'intégration manuelle des cartographies d'observations, sous forme de textures appliquées au modèle 3D. Par conséquent, ce projet n'offrant pas une interactivité directe, il s'est avéré nécessaire de développer un autre outil de gestion de données hétérogènes, dans le but premier de faciliter la compréhension de l'évolution des altérations et leur répartition spatiale sur de grandes surfaces tridimensionnelles telles que les peintures murales. L'expérience de NUBES et les différents programmes de recherche qui ont suivi (voir encart) ont fait émerger la nécessité d'un outil simple, interactif et plus largement accessible permettant de rassembler la documentation et d'y ancrer dynamiquement les observations.

C'est dans ce contexte et pour répondre à cette problématique qu'a été conçu et développé Aïoli $^{12}$, un outil en ligne d'annotation sémantique 3D pour la documentation d'objets patrimoniaux. Il s'agit de la première plateforme collaborative au stade de prototype opérationnel (www.aioli.cloud) ayant pour vocation de réunir les acteurs et experts du patrimoine culturel autour des nouvelles pratiques numériques, pour enrichir notre connaissance des objets patrimoniaux. Sur la base d'un jeu d'images photogrammétriques, la plateforme génère une représentation hybride $2 \mathrm{D}$ et $3 \mathrm{D}$, pouvant être enrichie par des annotations sémantiques ou des ressources complémentaires liées à l'objet (références textuelles, images d'archives, résultats d'analyses, rapport...) automatiquement propagées sur l'ensemble des images. Pour chaque objet patrimonial numérique, les utilisateurs peuvent définir et créer librement par une structure de description multicalque, générant simultanément une base de données multidimensionnelle qui rassemble et lie à l'objet physique étudié, les informations, les observations, les analyses afférentes. Cette approche conduit à mettre en place un continuum d'informations entre l'objet et les données hétérogènes produites par le réseau d'acteurs qui l'observe, l'étudie ou œuvre pour sa conservation et sa restauration, créant ainsi une sorte d'épiderme numérique. La dimension temporelle des objets et la nature multimodale des données collectées sont également prises en compte, pour permettre un suivi de l'état de conservation et les dégradations éventuelles. Aïoli propose donc de mettre en place un écosystème numérique qui vise à moyen terme à corréler de multiples annotations et données sous-jacentes sur le plan spatial, temporel et sémantique en exploitant notamment les thesauri, les vocabulaires contrôlés et les 
ontologies de domaine pouvant superviser des capacités nouvelles de méthodes d'apprentissage (intelligence artificielle) appliquées aux sciences patrimoniales. La mise à disposition de ce service repose sur deux évolutions technologiques majeures: le développement des techniques de photogrammétrie incrémentale, qui permettent de calculer dynamiquement un modèle 3D et la possibilité de réunir, de traiter massivement et de partager des données via une infrastructure cloud accessible par une interface web multiplateforme.

Cet outil, actuellement en phase de beta testing à l'échelle nationale et ancré dans l'essor des sciences participatives, vise à faire naître de nouvelles méthodologies de travail pluridisciplinaire, et à faire émerger de nouveaux scénarios d'analyse comparative et coopérative des objets patrimoniaux (fig. 8).

Figure 8

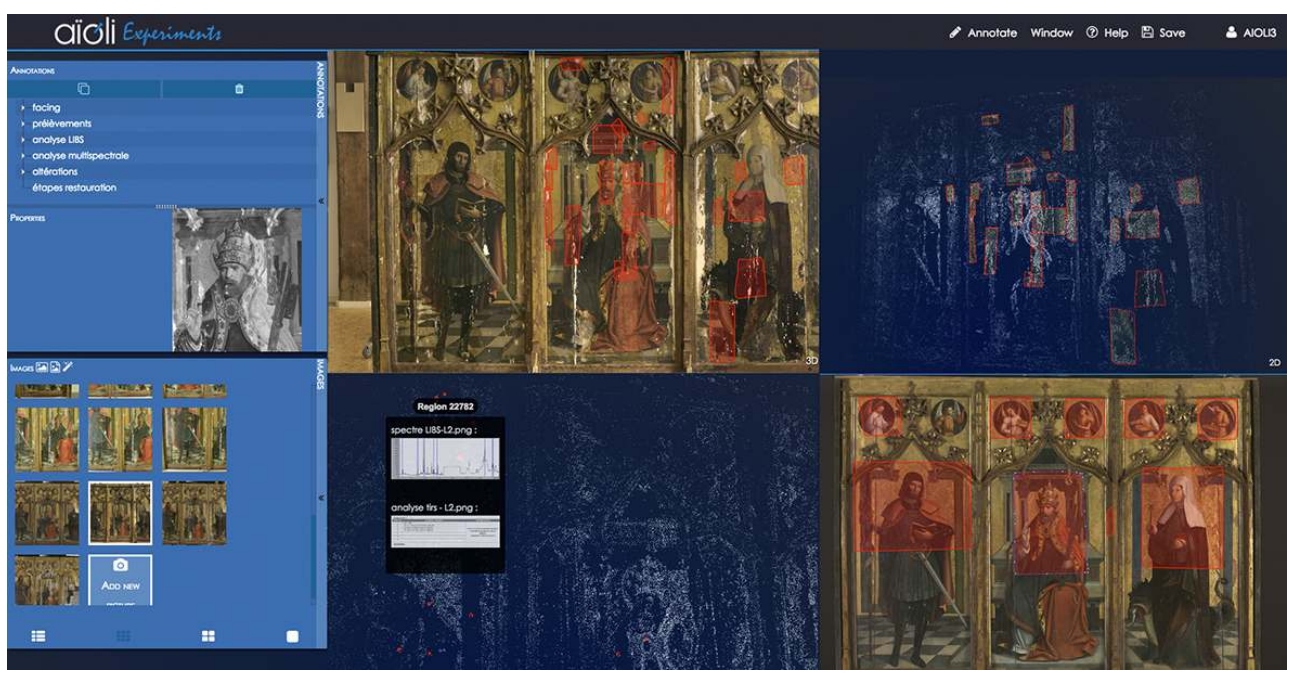

Exemple de projet Aïoli et de description multicalques sur le triptyque de Venasque (Vaucluse) (un des cas d'étude du projet FIAT LUX, ayant fait l'objet du reportage CNRS Image « Peintures en pixels »).

Phot. Anthony Pamart, 2018. (C) UMR MAP 3495 CNRS/MCC

\section{Perspectives et conclusion}

L'ensemble des développements et travaux menés en imagerie technique et sur l'intégration dans des plateformes collaboratives d'annotation sémantique 3D a conduit le Labcom MAP-CICRP à conduire de nouvelles réflexions sur les techniques de prises de vue, tant dans leur mise en œuvre que pour une accessibilité accrue aux nonprofessionnels de la conservation-restauration. La maîtrise et la diffusion de ces nouveaux supports d'imagerie numérique - image composite fausse couleur, cartographie multicalque, représentations hybrides 2D/3D - pourraient par ailleurs susciter une meilleure intégration dans les cursus de formation pour une exploitation optimale. L'imagerie numérique permet une production plus facile de documents et devient aujourd'hui l'accompagnement scientifique non destructif le plus accessible d'une opération de conservation-restauration. La fonctionnalité de ce panel d'outils (imagerie technique par photogrammétrie numérique et plateforme d'annotation sémantique 3D) est en mesure d'intéresser les professionnels de la conservation. Elle peut modifier leur approche sur le terrain grâce à sa capacité de mise en synergie de résultats provenant de 
différentes techniques d'analyse, de l'imagerie technique et la documentation acquise par le passé. Ces outils vont conduire les scientifiques spécialisés dans la conservation à mettre en cohérence leurs observations, leurs conclusions quant aux analyses faites à différentes échelles. Ils pourront, pour une zone donnée, jouer sur différentes segmentations des domaines de longueur d'ondes et leurs associations, les mettre en relation avec les analyses physiques et physico-chimiques : penser autrement l'utilisation de l'imagerie technique. Le responsable du bien culturel comme le restaurateur pourront apporter aux dossiers d'œuvres une dimension et des indications scientifiques.

Ce nouveau support et les données qu'il véhicule inscrivent la restauration non plus dans un moment circonscrit de l'histoire matérielle de l'œuvre mais dans une dynamique temporelle de suivi intégrant les données de la restauration, éventuellement de son histoire passée et permettant de suivre l'évolution du bien en documentant prochainement ses conditions environnementales.

De nouvelles potentialités de la plateforme Aïoli et de l'approche de fusion de données par l'image pourront être intégrées à ce support et vont concerner :

34 - la capacité à analyser plus finement la matérialité d'une œuvre et certaines de ses caractéristiques (compréhension de modifications locales de comportement ou de nature de matériau, forme et nature d'une dégradation...) par le biais d'un changement d'échelle et de la possibilité d'exploration ponctuelle d'un nuage de points à densité variable ;

- l'automatisation de la quantification métrique des données par la fusion des nuages de points obtenus par photographie numérique ou numérisée et issus d'outils de type scanner-laser ;

- l'exploitation des données multibandes issues d'acquisitions par caméra hyperspectrale, par thermographie IR ou radiographie.

37 L'imagerie numérique, dans sa dimension "plateforme collaborative », cristallise ainsi la dimension interdisciplinaire de la conservation-restauration, avec la mise en place aujourd'hui encore expérimentale, demain très commune - d'un "dossier de bien culturel » regroupant l'ensemble des données de connaissance historiques, archivistiques et de l'histoire matérielle du bien. L'utilisation de ces outils partagés par les architectes, les historiens, les conservateurs, les scientifiques, les restaurateurs va, de ce fait, conduire à une meilleure synergie interprofessionnelle. Elle concrétise, par ses possibilités et ses champs d'utilisation, toutes les composantes de la notion de conservation-restauration définie à la convention triennale de l'ICOM à New Delhi en 2011.

38 Par toutes ces capacités et potentialités, elle participera à une meilleure transmission des connaissances et savoirs acquis et facilitera in fine l'accès du public à l'information produite.

\section{ENCART : L'impulsion des programmes de recherche LABCOM}

Plusieurs programmes de recherche ont été initiés dans le cadre de la collaboration entre le MAP et le CICRP, entrant dans le cadre de programmes internes au MAP comme le programme NUBES ou dans le cadre de projets de recherche financés suite à un appel à projet national (Monumentum, FIAT LUX, SUMUM). Les objectifs de ces projets ont été de 
définir des méthodes et de concevoir des prototypes d'outils informatiques accessibles depuis le laboratoire jusqu'au terrain, utilisables par différents acteurs de la conservation sans qu'il soit nécessaire d'acquérir des infrastructures ou des équipements informatiques importants. Se basant sur les potentiels démontrés de l'imagerie technique numérique, ils constituent un enjeu majeur pour la conservation-restauration tant dans sa dimension curative que préventive. L'enjeu est d'autant plus important que ces outils, en réseau partagé, renforcent la vocation interdisciplinaire de la conservation des biens culturels. Ils seront aussi, à court terme, accessibles et facilement utilisables par tous les acteurs de la discipline.

\section{MONUMENTUM : MODÉLISATION NUMÉRIQUE ET GESTION DE DONNÉES POUR LA CONSERVATION DES STRUCTURES MAÇONNÉES (projet ANR « contenus numériques et interactions ", 2014-2017)}

Ce programme de recherche a permis de concevoir et d'initier le développement d'une plateforme logicielle prototype ouverte et extensible (devenue Aïoli) visant à faciliter la capitalisation et la gestion de connaissances pour la conservation matérielle de biens culturels ${ }^{13}$. Cette plateforme permet, dans une approche multi-échelle, la mise en relation de données variées décrivant les états du monument (données de relevé et d'imagerie technique, cartographies des détériorations, collectes photographiques, archives historiques, documents d'analyse, etc.). Elle s'appuie en particulier sur l'avancement simultané du projet FUI Culture 3D Cloud ${ }^{14}$ qui a permis l'automatisation des processus de photogrammétrie en calcul déporté et sur l'aboutissement des travaux menés lors du programme de recherche FIAT LUX qui a rendu possible la fusion des informations (voir ci- dessous). Sur le plan de la modélisation informatique, deux aspects innovants ont ainsi été explorés : d'un côté, l'idée de relier (et de rapprocher) la phase d'acquisition des données spatiales à celle de leur analyse et de leur interprétation; de l'autre côté, l'ambition d'élaborer des supports d'analyse (morphologie de l'édifice, état des surfaces, comportement des structures) interconnectés par un système de caractérisation sémantique basé sur des mécanismes de distribution/propagation (multi-échelles et multi-projections) de concepts structurés au sein d'une ontologie de domaine ${ }^{15}$. La recherche, menée pour la première fois dans le domaine de la conservation sur le développement de cette ontologie, a abouti à la mise en place de certains concepts qui seront intégrés à terme dans le moteur de recherche associé à Aïoli.

Coordinateur : L. De Luca (UMR 3495-MAP), CICRP (Marseille), PRISME-FRE 3520 (Orléans), École nationale des sciences géographiques (Saint-Mandé), Laboratoire de Mécanique et Génie civil - UMR 5508 (Montpellier), CRMH de la DRAC PACA (Aix-en-Provence)

Site web : http://www.monumentum.map.cnrs.fr/

\section{FIAT LUX : FUSION D'IMAGERIE ET SUIVI TEMPOREL POUR L'ÉTUDE DE PEINTURES À GÉOMÉTRIE COMPLEXE (Projets interdisciplinaires CNRS « Défi Imag'In », 2015-2017)}

43 Ce projet avait pour but de rendre possible la fusion d'images issues de la photodocumentation technique et des données physiques et physico-chimiques des matériaux. 
En abordant la spatialisation 3D de ces informations pour produire une documentation numérique pour la conservation des peintures du patrimoine, le projet avait pour ambition de lever certains verrous tels que la cohérence géométrique de différents capteurs, la possibilité d'une caractérisation spatialement localisée des matériaux ${ }^{16}$. Cette documentation dynamique permet d'accéder, d'explorer et de mettre en œuvre le suivi temporel des informations au sein de représentations interactives multidimensionnelles, depuis la plateforme prototype Aïoli.

Coordinateurs : L. De Luca (UMR 3495-MAP) \& J.-M. Vallet (CICRP), École nationale des sciences géographiques (Saint-Mandé), Laboratoire Électronique, Informatique et Image UMR 6306 CNRS/UB/Arts et Métiers/AgroSup (Dijon), Laboratoire d'Archéologie moléculaire et structurale - UMR 8220 CNRS-UMPC (Paris), LRMH USR 3224 CNRS/MCC/ MNHN (Champs-sur-Marne), CNR-ISTI (Pise, Italie)

Site web : http://www.fiatlux.gamsau.archi.fr

\section{SUMUM : STRATÉGIE DE DOCUMENTATION MULTIÉCHELLE, MULTIMODALE DU PATRIMOINE CULTUREL (Programme ANR 2017-2020)}

Dans la poursuite des problématiques et acquis issus des programmes MONUMENTUM et FIAT LUX, SUMUM appréhende les enjeux de conservation-restauration et de leurs acquisitions numériques (réflectance, multiéchelle...) d'œuvres hors normes, multiformes et dans des conditions environnementales maîtrisées - en intérieur «indoor »- et non maîtrisées - en extérieur « outdoor ».

Ces travaux vont s'appuyer sur trois approches complémentaires illustrées par les œuvres suivantes :

- Le suivi temporel post-restauration pour la tour Keith Haring, Paris, et celui d'une sculpture monumentale à morphologie complexe, exposée en extérieur : L'Arbre-serpent de Niki de Saint-Phalle, musée des Beaux-Arts d'Angers

- Le suivi temporel post-restauration d'une Expansion de César (musée d'Art contemporain, Marseille)

50 - La morphologie complexe et hors normes des œuvres indoor des Intégrations de la Fondation Vasarely (Aix-en-Provence) lors d'opérations de conservation-restauration programmées en 2018-2020 afin de concevoir des outils de suivi et d'aide à la décision.

51 Site web: http://anr-sumum.fr

\section{NOTES}

1. - BARAZZETTI, Luigi, REMONDINO, Fabio, SCAIONI, Marco, et al. « Geometric and radiometric analysis of paintings". International Archives of Photogrammetry, Remote Sensing and Spatial Information Sciences, 2010, vol. 38, Part 5. 
2. - LISSALDE, Claire. "L'image scientifique: définitions, enjeux et questions ». Bulletin des bibliothèques de France, 2001, vol. 46, $\mathrm{n}^{\circ}$ 5, p. 26-33.

3. - L'utilisation d'un capteur Sigma-Foveon composé de trois couches de photodiodes, respectivement sensibles au RVB, transposant en numérique la structure du film argentique, permet de restituer plus fidèlement la couleur mais ne reste exploitable qu'en faible sensibilité. Inversement, le capteur Fuji-XTrans, dérivant le module conventionnel de la " matrice de Bayer » généralement utilisé, permet une meilleure gestion du bruit même à haute sensibilité, pouvant par conséquent satisfaire certains contextes d'acquisition.

4. - DONDERO, Maria Giulia et REYES-GARCIA, Everardo. «Les supports des images: de la photographie à l'image numérique». Revue française des sciences de l'information et de la communication, 2016, $\mathrm{n}^{\circ} 9$. Voir le site : https://journals.openedition.org/rfsic/2124 [consulté le 01/04/2019].

5. - PAMART, Anthony, GUILLON, Odile, FARACI, Stéphan, et al. « Multispectral Photogrammetric Data Acquisition and Processing for Wall Paintings Studies». The International Archives of Photogrammetry, Remote Sensing and Spatial Information Sciences, 2017, vol. 42, p. 559.

6. - DONDERO, Maria Giulia et REYES-GARCIA, Everardo. «Les supports des images: de la photographie à l'image numérique ». Art. cit., et PAMART, Anthony, GUILLON, Odile, FARACI, Stéphan, et al. «Multispectral Photogrammetric Data Acquisition and Processing for Wall Paintings Studies ». Art. cit., p. 559.

7. - MANUEL, Adeline. Annotation sémantique 2D/3D d'images spatialisées pour la documentation et l'analyse d'objets patrimoniaux. Thèse de doctorat. Paris: École nationale supérieure d'arts et métiers-ENSAM, 2016.

8. - LI, Junfeng et WAN, Xiaoxia. « Superpixel segmentation and pigment identification of colored relics based on visible spectral image ». Spectrochimica Acta Part A: Molecular and Biomolecular Spectroscopy, 2018, vol. 189, p. 275-281.

9. - PETELER, Friederike, GATTET, Éloi, BROMBLET, Philippe, et al. « Analyzing the evolution of deterioration patterns: A first step of an image-based approach for comparing multitemporal data sets ». Dans Digital Heritage, 2015. S.l. : IEEE, 2015, p. 113-116.

10. - DE LUCA, Livio, BUSAYARAT, Chawee, STEFANI, Chiara, VÉRON, Philippe, FLORENZANO, M ichel. «A semantic-based platform for the digital analysis of architectural heritage ». Computers \& Graphics, 35, 2, 2011, p. 227-241. Voir le site : https://doi.org/10.1016/j.cag.2010.11.009 [consulté le 01/04/2019].

11. - VALLET, Jean-Marc, DE LUCA, Livio, FEILLOU, Marie, et al. « An interactive 3-dimensional database applied to the conservation of a painted chapel ». International Journal of Heritage in the Digital Era, 2012, vol. 1, n 2, p. 233-250 ; VALLET, Jean-Marc, DE LUCA, Livio, FEILLOU, Marie. «Une nouvelle approche spatio-temporelle et analytique pour la conservation des peintures murales sur le long terme ", In Situ [En ligne], 19|2012, mis en ligne le 24 septembre 2012, consulté le 31 mars 2019. URL: http://journals.openedition.org/insitu/9829 ; DOI : 10.4000/ insitu.9829.

12. - Voir le site : http://www.aioli.cloud/ [consulté le 07/06/2019].

13. - PETELER, Friederike, GATTET, Éloi, BROMBLET, Philippe, et al. « Analyzing the evolution of deterioration patterns... ». Art. cit., p. 113-116 ; VALLET, Jean-Marc, DE LUCA, Livio, FEILLOU, Marie, et al. «An interactive 3-dimensional database applied to the conservation of a painted chapel ». Art. cit., p. 233-250.

14. - Voir : http://wwwc3dc.fr.

15. - MESSAOUDI, Tommy, VÉRON, Philippe, HALIN, Gilles, et al. « An ontological model for the reality-based 3D annotation of heritage building conservation state ». Journal of Cultural Heritage, 2017.

16. - PAMART, Anthony, GUILLON, Odile, VALLET, J.-M., et al. «Toward a multimodal photogrammetric acquisition and processing methodology for monitoring conservation and 
restoration studies ». Dans Proceedings of the 14th Eurographics Workshop on Graphics and Cultural Heritage. Eurographics Association, 2016, p. 207-210.

\section{RÉSUMÉS}

L'imagerie scientifique est un outil incontournable des sciences de la conservation-restauration. La modification du support de la photographie, devenu numérique, le développement du rendu tridimensionnel qualitatif et quantitatif de l'image ont transformé la photographie technique en un outil d'investigation non destructif à part entière, indispensable et complémentaire des approches physiques et physico-chimiques classiques et en un outil-support de documentation. L'ensemble des développements et travaux menés en imagerie scientifique et sur l'intégration dans des plateformes collaboratives d'annotation sémantique 3D permet, outre une production plus facile de documents, de mettre en synergie les résultats provenant de différentes techniques d'analyse. Elle va modifier, dans un futur proche, l'approche sur le terrain des acteurs de la conservation et conduire à une meilleure synergie interprofessionnelle. Ils auront ainsi la possibilité de mieux mettre en cohérence leurs observations, leurs conclusions quant aux analyses faites à différentes échelles, dans une dynamique temporelle qui intègre les données de la restauration, de son histoire passée. À terme, et par l'intégration des données environnementales, ces plateformes seront un outil de suivi de l'évolution de l'état matériel du bien culturel et aussi de transmission des connaissances vers un public large.

Technical imaging has always been an essential tool in conservation science. Thanks to today's digital photography and its quantitative and qualitative tridimensional aspects, this imaging has become an autonomous and vital nondestructive instrument that complements classic physical and chemical analyses. It is also an essential tool for documentation purposes. Results coming from different techniques of analysis can now be synthesized by means of new developments in technical imaging and their insertion into collaborative 3D semantic annotation platforms. Technical imaging can modify the infield approach of conservation professionals and will help lead to better interprofessional dialogue in the future. Conservation professionals will have the assurance of better consistency in their observations, which can cover different time frames for restoration projects and historical data and conclusions drawn from analyses made at different scales. In the long term and after integration of environmental data, these platforms will become monitoring tools dedicated to following the state of conservation of cultural heritage objects and a tool for transmitting knowledge about these objects to a wider audience.

\section{INDEX}

Mots-clés : imagerie scientifique, traitement de l'image, fusion d'images, annotation sémantique, cartographie multicalque, base de données multidimensionnelle, multimodale et multispectrale, plateforme collaborative

Keywords : technical imaging, image processing, image fusion, semantic annotation, layered mapping, multidimensional multimodal and multispectral database, collaborative platform 


\section{AUTEURS}

\section{ROLAND MAY}

Directeur du Centre interdisciplinaire de conservation et de restauration du patrimoine (CICRP), Marseille Roland.May@cicrp.fr

ODILE GUILLON

Photographe, CICRP Odile.guillon@cicrp.fr

\section{ANTHONY PAMART}

Ingénieur d'études CNRS, UMR 3495 MAP CNRS/MC anthony.pamart@map.cnrs.fr

\section{JEAN-MARC VALLET}

Ingénieur de recherche, CICRP Jean-marc.vallet@cicrp.fr 